\title{
REVISÃO
}

\section{O PAPEL DA EDUCAÇÃO NUTRICIONAL NO COMBATE ÀS CARÊNCIAS NUTRICIONAIS}

\author{
THE NUTRITION EDUCATION ROLE IN \\ THE COMBAT AGAINST MICRONUTRIENT DEFICIENCIES
}

Rejane Andréa RAMALHO'
Cláudia SAUNDERS'

RESUMO

Embora a educação nutricional seja vista como um esforço destinado a mudar "hábitos alimentares", padrões alimentares são determinados por fatores que incluem, além de educação orientada para uma nutrição adequada, fatores socioeconômicos, ecológicos, culturais e antropológicos. Alguns destes fatores induzem à geração e manutenção de "tabus alimentares" que impedem, principalmente nas camadas mais carentes da população, a escolha adequada de alimentos para uma dieta balanceada. Este trabalho aborda algumas destas questões, numa tentativa de provocar reflexão sobre educação nutricional num contexto mais amplo como principal estratégia de combate à hipovitaminose $A$, considerada um dos mais importantes problemas nutricionais e de Saúde Pública do mundo atual. A principal causa desta carência é a ingestão inadequada de alimentos fonte de vitamina $A$, que muitas vezes está mais relacionada às questões culturais e hábitos alimentares do que a fatores econômicos. Vários estudos mostram que, no Brasil, os alimentos fonte deste nutriente são alvo de crenças, proibições e tabus. Conclui-se que o profissional de saúde deve ponderar os aspectos não só econômicos, mas também culturais envolvidos, principalmente quando a proposta de intervenção envolve educação nutricional.

Termos de indexação: vitamina A, hábitos alimentares, fatores socioeconômicos, tabu, educação nutricional.

\begin{abstract}
Nutrition education is viewed as a means of changing "food habits". However, dietary patterns depend on many other factors that cannot be overcome by appropriate nutrition teaching, such as socioeconomic, ecological, cultural and anthropological aspects. Some of these factors generate "alimentary taboos" which hamper the choice of foodstuffs for a balanced diet. This paper deals with some of these questions, in an attempt to increase awareness about nutrition education in a wider context as an effective strategy in the fight against vitamin A deficiency, one of the major Nutrition and Public Health problems of our world. The main determinant of this deficiency is inadequate nutrition generally unrelated to traditional "education" and socioeconomic factors. Several studies have shown that, in Brazil, the main cause of vitamin A deficiency is inadequate consumption of sources of the vitamin because of factors such as taboos, beliefs and bans. Health professionals must be made more aware that, when micronutrient deficiencies are involved, they must look not only at social or economic variables, but also at the cultural aspects involved.
\end{abstract}

Index terms: vitamin A, food habits, socioeconomic factors, cultural, taboo, nutrition education.

\footnotetext{
(1) Departamento de Nutrição e Dietética, Centro de Ciências da Saúde, Instituto de Nutrição Josué de Castro, Universidade Federal do Rio de Janeiro, Bloco J, 2o andar, Ilha do Fundão, 21944-970, Rio de Janeiro, RJ, Brasil. Correspondência para/Correspondence to: R.A. RAMALHO. E-mail: aramalho@rionet.com.br
} 


\section{INTRODUÇÃO}

Ao longo dos anos, os historiadores e cientistas descreveram o desenvolvimento das comunidades, assinalando a preocupação com a alimentação, disponibilidade de água e condições climáticas, apontados como fatores básicos para sobrevivência do homem (Canesqui, 1968).

Na literatura, os dados sobre o consumo alimentar como fenômeno psicossocial são fragmentados e discutidos por várias áreas da ciência. Muito se tem descrito sobre o consumo de alimentos de diferentes grupos sociais, associando "o que" as pessoas comem com suas características demográficas e estilos de vida, mas pouco se sabe sobre a razão para a seleção individual de alimentos (Rappoport et al., 1992).

Os hábitos alimentares e as necessidades nutricionais do homem contemporâneo começaram a ser estabelecidos no passado pré-histórico, e as práticas alimentares sofreram adaptações muitas vezes para hábitos pouco saudáveis, o que constitui desvantagem para a saúde, associando-se muitas vezes com os desvios ponderais e desenvolvimento de deficiências nutricionais múltiplas ou específicas (Canesqui, 1968; Garn \& Leonard, 1989) como a hipovitaminose A, cujas manifestações podem ocorrer sem sinais clínicos detectáveis ou sem estarem associadas a doenças multicarenciais claramente definidas, dificultando seu diagnóstico pela equipe de saúde (Ramalho, 1998).

Este trabalho tem como objetivo abordar algumas questões relacionadas aos aspectos socioeconômicos e culturais que interferem no consumo de alimentos fonte de vitamina A, visando subsidiar programas de intervenção, principalmente quando essa proposta envolver educação nutricional, como principal estratégia para o controle da hipovitaminose $\mathrm{A}$.

\section{ASPECTOS SOCIAIS E CULTURAIS DA ALIMENTAÇÃO}

$\mathrm{O}$ ato da busca, da escolha, do consumo e proibições do uso de certos alimentos dentre todos os grupos sociais são ditados por regras sociais diversas, carregadas de significados. Apreender a especificidade cultural dessas regras sociais, as quais precisam ser explicadas em cada contexto particular é de extrema importância, pois o alimento constitui uma linguagem (Daniel \& Cravo, 1989).

O alimento é algo representado, isto é, apreendido com significado cognitivo. Nem tudo que pode ser utilizado como alimento é percebido como tal. Ademais, o comer não satisfaz apenas a necessidade biológica, mas preenche também funções simbólicas e sociais. A comensalidade permeia todas as relações sociais de diferentes classes de uma mesma sociedade apresentando sempre uma dimensão cultural (Castro, 1941; Daniel \& Cravo, 1989).
O caráter simbólico do alimento também se diferencia com a idade, situação social e outras variáveis. Em todas as faixas etárias, encontra-se uma alimentação entendida como apropriada, variando a adequação em relação ao sexo e papéis sociais. Existe portanto, um processo de socialização que procura mostrar o comportamento alimentar mais apropriado a diferentes segmentos da sociedade. Entretanto, estas questões são permeadas pelo poder aquisitivo dos segmentos sociais e por oscilações entre comer aquilo que é ditado pela nossa cultura e aquilo que é entendido como saudável (Daniel \& Cravo, 1989).

\section{ASPECTOS ECONÔMICOS LIGADOS A ALIMENTAÇÃO}

A má nutrição não é problema só dos pobres. Os ricos - individuos e países - sofrem de obesidade, doenças crônicas, hipertensão arterial, câncer, diabetes mellitus, entre outras. A má nutrição dos pobres que era caracterizada pela magreza, nanismo e menor resistência às infecções (Dutra de Oliveira et al., 1996), atualmente tem sido modificada por crescente prevalência de excesso de peso (Monteiro et al., 1995; Pereira et al., 1998). Entretanto, a carência de micronutrientes pode se manifestar independentemente das condições socioeconômicas.

Inquéritos nutricionais têm apontado com freqüência a inadequação dos hábitos alimentares e do grau de conhecimento de nutrição de populações de baixa renda dentre os fatores determinantes da subnutrição, ao invés de considerá-los também como integrantes de uma situação na qual aparecem como resultado de normas estabelecidas e de condições criadas pelo sistema produtivo vigente (Canesqui, 1976; Instituto..., 1982; McAuliffe et al., 1991; Coelho et al., 1995), pois, sabe-se que a boa alimentação e boa nutrição dependem da produção e distribuição dos alimentos, que são influenciados pela economia do país e também pela educação da população (Dutra de Oliveira et al., 1996).

Outro aspecto da comensalidade das camadas de baixa renda no meio urbano é abordado por Zaluar (1980). Para estas, existem alimentos que são comida e outros que não são. Comida é basicamente feijão, arroz e carne. As verduras, os legumes e as frutas são citados como alimentos que servem para "tapear a fome".

Em trabalho desenvolvido com população carente, os alimentos são classificados em diferentes categorias como "alimentos fortes e fracos" "pesados e leves", "tem vitamina e não tem vitamina". A noção de alimento "forte" foi atribuída aos alimentos que tem sabor marcante e que "sustentam", já a noção de alimentos "fracos" foi atribuída aos alimentos que não sustentam nem tem gosto. A noção de alimentos "pesados" foi relacionada com alimentos que provocam reação no aparelho digestivo e considerados inadequados para algumas refeições; também são definidos 
como aqueles indicados para serem ingeridos durante o dia. São incluídos nesta categoria os alimentos "fortes", que foram relacionados com o fornecimento de energia ao organismo para o trabalho. Os alimentos "leves" foram definidos como aqueles que não fazem bem nem mal, adequados para pessoas doentes (Canesqui, 1976; Campos, 1982).

A noção de "vitamina" foi atribuída àqueles alimentos que fortificam o organismo e fazem bem para a saúde. Há menos vitaminas nos alimentos fracos, exceto frutas e verduras, sendo estes considerados como tendo vitaminas, mas não como "comida" que sustenta, pois, não conseguem saciar a fome. A alimentação foi ainda relacionada com uma função bastante ampla e imediata: "matar a fome, encher a barriga, sustentar o corpo, dar disposição pra trabalhar, pra cuidar da casa e das crianças" (Canesqui, 1976).

No Brasil os alimentos também são classificados em "quentes", sendo aqueles impróprios para o aparelho digestivo, e em "frios" que são aqueles impróprios para o aparelho respiratório, assim como há a interdição de se comer fruta fria com o corpo quente (Campos, 1982).

\section{TABUS ALIMENTARES}

A palavra tabu é de origem polinésia e significa alguma coisa que não pode ser definida. Alguma coisa que escapa ao nosso sentir de civilizados (Castro, 1941). Simboliza algo proibido e intocável (Mota \& Penna, 1991).

Tabu então é uma interdição, uma proibição categórica sem uma explicação racional. Interdição que não é ordenada por ninguém, mas que parece ser constituída por si mesma, sem nenhum fundamento, nem insinuação de lógica (Castro, 1941).

Cunha (1982) o define como "aquilo que por convenção ético-religiosa é proibido ou vulnerável", tornando o objeto relacionado ao tabu, excluído do uso cotidiano. A interdição não é motivada por justificativas explicáveis, a sanção temida ou aguardada em caso de violação do tabu não está prevista em leis, sendo apenas uma desgraça psíquica ou física.

Para Freud (1975) o tabu tem dois significados opostos, o do sagrado "sacer", que não pode ser tocado sem ser manchado, e o do inquietante, perigoso, proibido ou impuro. Para ele não existe racionalidade atrás dos tabus, que são empregados como forma de enfrentar o desconhecido e superar angústias e medos.

Já os polinésios podiam apreender o significado total, de acordo com esta lei da organicidade cultural que faz com que os indivíduos inseridos numa determinada cultura estejam aptos a compreender e decifrar suas concepções simbólicas. Assim sendo, o real sentido atribuído à palavra tabu pelos polinésios, nunca será entendido completamente pelo homem moderno ocidental.
Outras definições foram atribuídas ao termo entre diferentes povos, como romanos e gregos, confirmando que o tabu não é privilégio dos polinésios, e esteve presente na evolução de várias culturas, representando traço indispensável na estruturação moral de todos os grupos humanos (Peirano, 1977; Mota \& Penna, 1991; Spolidoro \& Spolidoro, 1991).

Alguns aspectos são considerados importantes na gênese do tabu como instinto do medo e do temor do desconhecido. Condensando essas forças em um determinado objeto, criar-se-ia o tabu, que aos poucos iria se desligando desses sentimentos e constituindo-se, então, em uma força independente, capaz de atuar por si mesmo, como algo ambivalente, divino e impuro, temido e desejado, objeto de admiração e de ódio (Castro, 1941).

Por outro lado, também podem ser vistos como elementos de harmonização das relações sociais e pessoais, tendo funções sociais, tais como ajudar o povo a refletir sobre si mesmo como uma comunidade à parte (Harris, 1978).

Um exemplo positivo de seu emprego ocorre entre os índios Guaiaqui funcionando como elemento estruturador, fundamento da sociedade. Acredita-se que é proibido ao caçador consumir carne de suas próprias presas, por acreditar que comer os animais mortos por eles próprios os farão incapazes de caçar novos animais. Assim, o produto de sua caça é distribuído e trocado com os outros membros da comunidade. Neste caso, se estabelece uma relação negativa entre o caçador e o produto de sua caça. Desta forma, todos os homens são colocados na mesma posição, e a reciprocidade do dom da alimentação mostra-se a partir daí não apenas possível, mas necessária. Os indivíduos são obrigados a se separarem de suas caças, e a confiar nos outros membros da sociedade, permitindo assim que o laço social se estabeleça. Os indivíduos perdem autonomia, mas a sociedade ganha força já que a separação do caçador de sua caça torna a união entre os caçadores mais forte (Clasters, 1988).

Deve-se diferenciar tabus alimentares e hábitos alimentares, sendo que os hábitos refletem os padrões culturais e socioeconômicos dominantes de uma determinada sociedade e mudam quando há modificação nesses padrões (Mota \& Penna, 1991).

É na ação das pessoas no momento da escolha de um determinado alimento em detrimento de outros, que se pode verificar o grau de comprometimento que eles têm com padrões culturais (costumes regionais, tradições familiares, crenças, hábitos e tabus), capazes de impedir que alimentos existentes em abundância nas comunidades sejam consumidos (Trigo, 1989).

\section{TABUS E HÁBITOS ALIMENTARES NO BRASIL}

No Brasil restam vestígios de tabus alimentares em nossos costumes, sobreviventes de nossa organização 
cultural. O hábito alimentar brasileiro é um reflexo dos padrões sócio-culturais introduzidos pela imigração maciça de diferentes grupos étnicos no Brasil, causando diferenças em certas áreas ou regiões do país (Dutra de Oliveira et al., 1996). Assim esfacelados, encontram-se pelo Brasil algumas superstições e proibições quanto ao consumo de alimentos, isoladamente, ou em misturas, em certas etapas da vida, ou em determinados horários do dia, entre outros (Harris, 1978; Backes-Clement, 1988).

Estas superstições alimentares não são, entre nós, tão abundantes quanto em outros países como Austrália, Polinésia e África Oriental, onde para os habitantes dessas regiões, a ingestão alimentar é sempre controlada por inúmeros tabus, acompanhada de um complicado ritual e de um perigo especial, porque a "alma pode escapar pela boca, ou um inimigo presente pode arrancá-la com artifícios mágicos". Estas superstições se explicam, porque nestas regiões imperam as organizações totêmicas. É certo que na nossa formação étnica entraram indivíduos dessas áreas culturais - negros de tribos totêmicas da África Ocidental. Mas estes negros, arrancados brutalmente de sua terra, que tiveram suas raízes a esta terra quebradas violentamente, não mantêm suas tradições no novo habitat com a mesma força original (Harris, 1978; Backes-Clement, 1988).

Esses fenômenos não sobrevivem inteiramente a uma mudança de habitat: ou perdem o sentido de vida e morrem, ou, quando muito, ficam vegetando enxertados nos troncos de manifestações mais intensas de vida regional, para onde foram levados. Foi isto o que aconteceu com a organização totêmica dos negros batunos trazidos à América (Castro, 1941).

O panorama alimentar no Brasil é complexo e peculiar de cada região, permeado de superstições, tabus e hábitos alimentares de diferentes origens: negras, índias, portuguesas que se coloriram de um tom diferente sob os aspectos múltiplos das variantes regionais.

É importante lembrar que em uma sociedade, a proibição ou prescrição de alimentos, segundo a teoria popular, repousa numa certa observação e experimentação, de maneira que, apesar de se diferenciarem dos modelos científicos oficiais, não devem ser consideradas irracionais ou desprovidas de lógica e de consistência interna, mas devem ser captadas pela riqueza que contêm e vistas como possibilidades de encaminhar soluções para os problemas nutricionais.

\section{PENSANDO A QUESTÃO DA HIPOVITAMINOSE A}

A carência de vitamina A é considerada um dos maiores problemas nutricionais em Saúde Pública no Brasil (Flores et al., 1983; Carvalho et al., 1995; Assis et al., 1997). A epidemiologia da deficiência deste nutriente tem sido geralmente bem estudada não apenas nos aspectos biológicos, como também nos seus fatores determinantes
(Underwood, 1978; Organización..., 1982; Campos et al., 1987; Fawzi et al., 1993; Glasziou \& Mackerras, 1993; Underwood, 1993; Sommer, 1995). Com exceção das situações de extrema pobreza, a renda e escolaridade parecem não ter relação na determinação desta doença carencial, reforçando a tese de que a ingestão inadequada de alimentos fonte de vitamina A seja o principal fator etiológico da carência desta vitamina e que sua exclusão ou baixo consumo estão mais relacionados a questões culturais e hábitos alimentares do que a fatores econômicos (Brünken \& Flores, 1993; 1994; Coelho et al., 1995). Tal constatação aponta para o aumento do consumo de alimentos fonte de vitamina A como a principal estratégia, a longo prazo, no combate à hipovitaminose $\mathrm{A}$ em nível mundial.

Alguns estudos têm demonstrado a associação entre fatores relacionados a tabus e a exclusão de alimentos importantes, como os de fonte de vitamina A, principalmente os de origem vegetal, considerados como os mais acessíveis do ponto de vista financeiro (Castro, 1941; Mota \& Penna, 1991). Vários autores alertam que esforços no sentido de aumentar a ingestão total de alimentos não necessariamente aumentarão o consumo de vitamina $A$, devido a fraca relação entre a ingestão da vitamina em questão e a ingestão energética total da dieta, baseando-se na análise da densidade de vitamina $A$ e de energia nas dietas analisadas pelo Estudo Nacional de Despesa Familiar (ENDEF) 1974-1975 (Instituto..., 1982). Via de regra o que se observa é o aumento quantitativo da dieta consumida habitualmente pela população sendo preciso então, a mudança na sua estrutura (Batista-Filho et al., 1971; Instituto..., 1982).

O uso de vegetais na alimentação do brasileiro é reflexo dos padrões culturais. Os índios brasileiros não davam importância aos vegetais verdes. O uso de vegetais, característicos da cozinha africana, foi introduzido no Brasil pelos negros escravizados (Freire, 1987). Tanto é assim que no sertão brasileiro, onde foi menor a influência negra, o consumo de vegetais na alimentação é reduzido.

A base da dieta tradicional do Nordeste - arroz, feijão e farinha de mandioca - é extremamente pobre em vitamina A (McAuliffe et al., 1991). Os inquéritos alimentares em Pernambuco (Batista-Filho et al., 1971; Deus et al., 1979), comprovaram que a vitamina A, entre todos os nutrientes estudados, é o que tem os mais baixos índices de adequação, com apenas 20\% das crianças atingindo as recomendações. Em outras regiões do país também foi encontrada adequação muito baixa no consumo de vitamina A com resultados variando entre 10 e 25\% de adequação (Roncada, 1972; Instituto..., 1982; Trigo, 1985).

Trabalhos realizados em diferentes regiões do Brasil revelam que os alimentos fonte de vitamina $A$ são alvo de várias crenças, proibições e tabus alimentares, e que muitas vezes estão relacionados a momentos fisiológicos de grande importância, sob o ponto de vista nutricional, 
tais como: gestação, lactação, desmame e os primeiros anos de vida da criança (Trigo, 1989; Brünken \& Flores, 1993; 1994; Ramalho et al., 1994; Coelho et al., 1995). Chama-se atenção para o fato de que altas cifras de morbi-mortalidade são observadas nesses momentos biológicos, e que eles constituem fatores de risco para diferentes enfermidades carenciais, sobretudo para a vitamina $A$ (Underwood, 1993).

A literatura é abundante em relação às restrições que afetam o consumo de fontes vegetais, que não têm nenhum fundamento biológico sendo sobrevivência cultural das interdições dos senhores a seus escravos e outros indivíduos (Castro, 1941; Brünken \& Flores, 1993; 1994). De acordo com Castro (1974), no clássico livro "Geografia da Fome", esses tabus tornaram-se verdadeiras barreiras psicológicas contra o uso de vegetais na alimentação, fazendo com que as pessoas perdessem o gosto por esses e se desinteressassem por completo pelo seu cultivo. Com isso acentuou-se a utilização dos carboidratos na alimentação, com o excesso de açúcar desequilibrando as funções metabólicas.

O baixo consumo ou a exclusão dos alimentos fonte de vitamina A reconhecidamente associados a fatores culturais e hábitos alimentares, são achados importantes e servem para provocar uma reflexão sobre a prática da educação nutricional no país tendente a desenvolver técnicas que conduzam a modificações de práticas alimentares, principalmente dos grupos de risco (Brünken \& Flores, 1993; Coelho et al., 1995).

O grupo chave para as modificações das práticas dietéticas nas famílias são as mulheres, principalmente as que se encontram em idade reprodutiva visto que geralmente são elas que controlam as práticas alimentares da família, principalmente nos períodos de aleitamento, desmame e alimentação da crianças em idade pré-escolar (Underwood, 1993).

Promover o aumento do consumo de alimentos ricos em vitamina A, sobretudo os de origem vegetal, pode ser um exercício inútil se não forem solucionadas as razões para o não consumo. Para modificar a estrutura da dieta consumida principalmente por aqueles com maior risco de deficiência, são necessárias novas técnicas de comunicação, como por exemplo o estabelecimento de um plano de marketing como via de suprir informações ao público alvo.

A utilização da prática de transferir informações aos formadores de opinião como profissionais envolvidos com veículos de comunicação de massa, tem-se mostrado um excelente canal para mudanças desejáveis no comportamento da população, devendo, portanto, serem acionados mecanismos que promovam tal articulação. Tais ações seriam reforçadas com programas de Educação em Saúde e Nutrição que contemplem informações a partir da pré-escola até o $2^{\mathrm{O}}$ grau e com maior aprofundamento das questões nutricionais no nível universitário, visando a modificação de hábitos alimentares para o controle de carências nutricionais.

\section{CONSIDERAÇÕES FINAIS}

A seleção de alimentos é muito complexa e influenciada por muitos outros fatores além do acesso aos alimentos e o conhecimento de nutrição. Embora saiba-se que quando os alimentos não estão disponíveis é bem provável que ocorra deficiência, por outro lado, a abundância não assegura ótima nutrição devido ao componente comportamental que determina a escolha dos alimentos.

O profissional de saúde ao sugerir qualquer intervenção na área alimentar deve ponderar os aspectos não só econômicos mas também os culturais envolvidos, principalmente quando a proposta de intervenção envolve aspectos educativos. A modificação das práticas alimentares e as modificações da qualidade da dieta como estratégia de combate a carência de vitamina A na população, é de extrema importância e deve ser objeto de reflexão para que as ações educativas propostas possam se tornar elemento efetivo de transformação de hábitos alimentares inadequados.

\section{REFERÊNCIAS BIBLIOGRÁFICAS}

ASSIS, A.M., PRADO, M.S., FREITAS, M.C.S., CRUZ, M.M. Deficiência de vitamina A e desnutrição-energético protéica em crianças da localidade do Semi-árido Baiano. Revista de Nutrição da PUCCAMP, Campinas, v.10, n.1, p.70-78, 1997.

BACKES-CLEMENT, C. Antropologia e psicanálise. In: COPANS, J., TORNAY, S., GODELIER, M., BACKES-CLEMENT, C. Antropologia, ciência das sociedades primitivas? Lisboa : Edições 70, 1988. p.213-238.

BATISTA-FILHO, M., CHAVES, N., VARELA, R.M., SOUZA MARTINS, M.H, SALZANO, A.C., BAZANTE, M.O., GOMES TEIXERA, S.M., LIMA, E.J.C., REIS, F.M., MARTINS, G.C., LINHARES, E.R. Inquérito nutricional em área urbana da Zona das Mata do Nordeste Brasileiro - Água Preta - Pernambuco. $O$ Hospital, v.79, n.5, p.139-155, 1971.

BRÜNKEN, G.S., FLORES, H. Consumption of vitamin A rich foods. Xerophtalmia Club Bulletin, London, v.54, p.3-4, 1993.

BRÜNKEN, G.S., FLORES, H. Why do diets lack vitamin A. Nutriview, v.3, p.1-3, 1994.

CAMPOS, F.A.C.S., FLORES, H., UNDERWOOD, B. Effect of an infection on vitamin A status of children as mensured by the Relative Dose Response (RDR). American Journal of Clinical Nutrition, Bethesda, v.46, n.1, p.91-94, 1987.

CAMPOS, M.S. Poder, saúde e gosto: um estudo antropológico acerca dos cuidados possíveis com a alimentação e o corpo. São Paulo : Cortez, 1982. 130p.

CANESQUI, A.M. Antropologia e alimentação. Revista de Saúde Pública, São Paulo, v.20, n.3, p.207-216, 1968.

CANESQUI, A.M. Comida de rico, comida de pobre: um estudo sobre alimentação num bairro popular. Campinas, 1976. 267p. Tese (Doutorado em Ciências ) - Faculdade de Ciências Médicas, Universidade Estadual de Campinas,1976. 
CARVALHO, C.M.G., FARFAN, J.A., WILKE, B.C., VENCOVSKY, R. Prevalência de hipovitaminose A em crianças da periferia do Município de São Paulo, Brazil. Caderno de Saúde Pública, Rio de Janeiro, v.11, n.1, p.85-96, 1995.

CASTRO, J. Fisiologia dos tabus. 2.ed. Rio de Janeiro : Nestlé, 1941.62p.

CASTRO, J. Geografia da fome: o dilema brasileiro: pão ou aço. 10.ed. Rio de Janeiro : Antares, 1974. 316p.

CLASTERS, P. O arco e o cesto. In: CLATERS, P. A sociedade contra o Estado. 4.ed. Rio de Janeiro : Francisco Alves, 1988. p.80-81.

COELHO, C.S.P., RAMALHO, R.A., ACCIOLY, E. O inquérito dietético na avaliação do estado nutricional de vitamina A em gestantes. Ars Cvrandi Clínica Médica, Rio de Janeiro, v.6, n.28, p.44-60, 1995.

CUNHA, A.S. Dicionário etimológico Nova Fronteira da língua portuguesa. Rio de Janeiro : Nova Fronteira, 1982. p.748-749.

DANIEL, J.M.P., CRAVO, V.Z. O valor social e cultural da alimentação. Boletim de Antropologia, v.2, n.4, p.70-83, 1989.

DEUS, M.B., COELHO, H.A.L., ROMANI, S.A.M. Consumo alimentar de Pernambuco. Recife : UFPE. 1979. 31p.

DUTRA DE OLIVEIRA, J.E., CUNHA, S.F.C., MARCHINI, J.S. A desnutrição dos pobres e dos ricos: dados sobre a alimentação no Brasil. São Paulo : Sarvier, 1996. 123p.

FAWZI, W.W., CHALMERS, T.C., HERRERA, M.G., MOSTELLER, F. Vitamin A supplementation and child mortality: a metaanalisis. JAMA, Chicago, v.269, n.7, p.898-903, 1993.

FLORES, H., CAMPOS, F.A.C.S., UNDERWOOD, B. Importance of the early diagnoses of vitamin A deficiency at the epidemiological level. International Journal for Vitamin and Nutrition Research, Bern, v.24, p.23S-24S, 1983. Supplement.

FREIRE, G. Açúcar: em torno da etnografia, da história e da sociologia do doce no Nordeste Brasileiro. 3.ed. Recife : Massangana, 1987. 213p.

FREUD, S. Totem y tabu. Madrid : Alianza Editorial, 1975. p.2930.

GARN, S.M., LEONARD, W.R. What did our ancestors eat? Nutrition Reviews, New York, v.47, n.11, p.337-345, 1989.

GLASZIOU, P.P., MACKERRAS, D.E.M. Vitamin A supplementation in infectious diseases: a meta-analysis. British Medical Journal, London, v.306, n.6874, p.366-370, 1993.

HARRIS, M. Vacas, porcos, guerras e bruxas: os enigmas da cultura. Rio de Janeiro : Civilização Brasileira, 1978. 205p.

INSTITUTO BRASILEIRO DE GEOGRAFIA E ESTATÍsTICA. Perfil estatístico de crianças e mães no Brasil: aspectos nutricionais, 1974-75. Rio de Janeiro, 1982. p.185-211.

McAULIFFE, J., SANTOS, L.M., DINIZ, A.S., BATISTA-FILHO, M., BARBOSA, R.C.C. A deficiência de vitamin A e estratégias para o seu controle: um guia para as Secretarias Municipais de Saúde. Fortaleza : Project HOPE, 1991. 29p.

MONTEIRO, C.A., MONDINI, L., SOUZA, A.L.M., POPKIN, B.M. Da desnutrição para a obesidade: a transição nutricional no Brasil. In: MONTEIRO, C.A. Velhos e novos males da saúde no Brasil: a evolução do país e de suas doenças. São Paulo : Hucitec, 1995. p.251.
MOTA, J.A.C., PENNA, F.J. Tabus alimentares. In: WEHBA, J. et al. Nutrição da criança. Rio de Janeiro : Fundo editorial BYK, 1991. p.257-268.

ORGANIZACIÓN MUNDIAL DE LA SALUD. Prevención y tratamiento de la carencia de vitamin A y de la xeroftalmia. Ginebra, 1982. 74p. (Série Informes Técnicos, 672).

PEIRANO, M.G.S. Proibições alimentares numa comunidade de pescadores. Brasília, 1977. 165p. Dissertação (Mestrado em Antropologia Social) - Universidade de Brasília, 1977.

PEREIRA, R.A. Avaliação antropométrica do estado nutricional. In: SICHIERI, R. Epidemiologia da obesidade. Rio de Janeiro : EDUERJ, 1998. p.62-63.

RAMALHO, R.A. Deficiência marginal de vitamina A em puérperas, recém-nascidos e pré-escolares atendidos em três Serviços de Saúde do Município do Rio de Janeiro. Rio de Janeiro, 1998. 76p. Tese (Doutorado em Saúde Pública) - Escola Nacional de Saúde Pública, Fundação Oswaldo Cruz, 1998.

RAMALHO, R.A., MARTINS, C.S.F., MONTEIRO, R.C.A., FERREIRA, R.M. Situação atual da hipovitaminose A e os recentes avanços em diagnóstico e prevenção. Clínica Pediátrica, São Paulo, v.1, n.18, p.11-14, 1994.

RAPPOPORT, L.H., PETERS, G.R., HUFF-CORZINE, L., DOWNEY, R.G. Reasons for eating: an exploratory cognitive analysis. Ecology of Food and Nutrition, London, v.28, n.3, p.171-189, 1992.

RONCADA, M.J. Hipovitaminose A: niveis séricos de vitamina A e caroteno em populações litorâneas do Estado de São Paulo, Brasil. Revista de Saúde Pública, São Paulo, v.6, n.1, p.3-18, 1972.

SOMMER, A. Vitamin A deficiency and its consequences: a field guide to detection and control - Epidemiology. 3.ed. Geneva : World Health Organization, 1995. 65p.

SPOLIDORO, A. SPOLIDORO, M.N. Tabus alimentares. In: WEHBA, J. et al. Nutrição da criança. Rio de Janeiro : Fundo Editorial BYK, 1991. p.268-272.

TRIGO, M. Análise de situação alimentar de dois núcleos populacionais de Marabá, Pará. Alimentação, São Paulo, v.80, n.5, p.17-27, 1985.

TRIGO, M. Tabus alimentares na Região do Norte do Brasil. Revista de Saúde Pública, São Paulo, v.23, n.6, p.455-464, 1989.

UNDERWOOD, B.A. Estrategias a largo plazo para el control de las deficiencias de micronutrientes. Arlington, Virginia: USAID, 1993. p.70-76. (Vitamin A Field Support Project (VITAL) nIN-14).

UNDERWOOD, B.A. Hypovitaminosis A and its control. Bulletin of the World Health Organization, Geneva, v.56, n.3, p.525$541,1978$.

ZALUAR, A. As mulheres e a direção do consumo doméstico. Rio de Janeiro : FINEP, 1980. v.4. (Relatório final da Pesquisa Ciências Sociais e Nutrição).

Recebido para publicação em 13 de maio de 1998 e aceito em 12 de março de 1999. 